\title{
STROKE HEALTH: A DIGITAL TOOL TO SUPPORT SHARED DECISION-MAKING AFTER STROKE
}

\author{
Emma K Kjörk, Gunnel Carlsson, Åsa Lundgren-Nilsson and Katharina S Sunnerhagen
}

Institute of Neuroscience and Physiology, Department of Clinical Neuroscience and Rehabilitation, at the Sahlgrenska Academy,

University of Gothenburg, Sweden

\section{Conclusions}

- Stroke Health has the potential to identify post-stroke problems and support shared decisionmaking for persons living with stroke.

- It is of importance to involve the users in the design process to be able to offer a user-friendly tool built on patients' needs.

\section{Background}

Difficulties to understand health-information and describing needs after stroke can lead to a risk of not receiving adequate support.

Stroke Health is an assistive digital tool developed to support shared decision-making after stroke. Stroke Health comprises self-report on basis of the eleven problem areas in the Post-Stroke Checklist and stroke-related information.

\section{Purpose}

The aim was to explore user-needs to enable development of a digital tool to support shared decision-making.

\section{Methods}

A prototype of the digital tool called Stroke Health was developed according to service-design thinking including rapid prototyping.

- Stroke Health was tested by 12 persons with prior stroke (median 65 years (43-85), 5 women) recruited from the Stroke Association and an out patient rehabilitation center.

- Three focus-group discussions were performed regarding user-needs and possible improvements of Stroke Health.

- Data analyses are ongoing. Patterns and themes derived from the focus group discussions will serve as guidance for further development of Stroke Health.

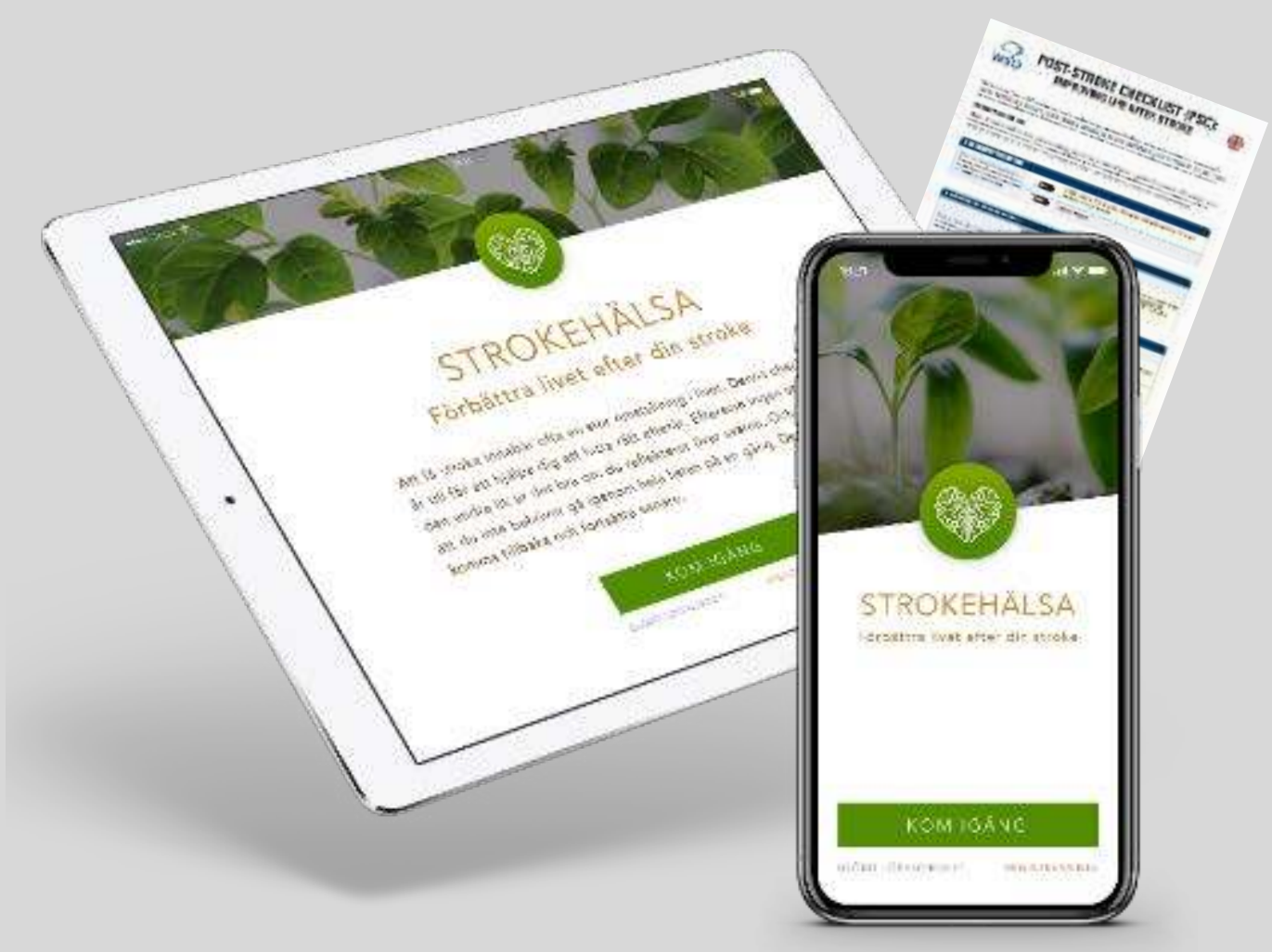

Preliminary results

- The knowledge that post-stroke problems faced by the patient are common helps them to normalize their situation.

- Stroke Health was expected to enable better communication between clinicians and patients.

- The users found Stroke Health easy to use but offered suggestions to improve design and information.

- The risk of not capturing discrete problems by using Stroke Health were mentioned and possible amendments were discussed.
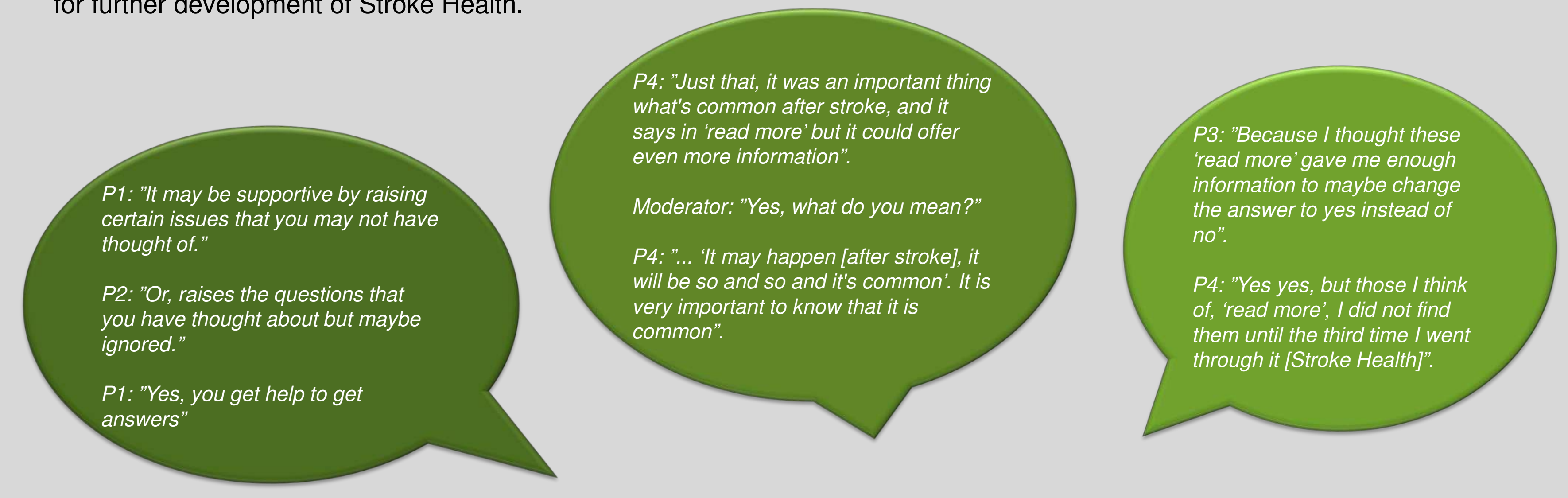

\section{SAHLGRENSKA ACADEMY}

Institute of Neuroscience and Physiology
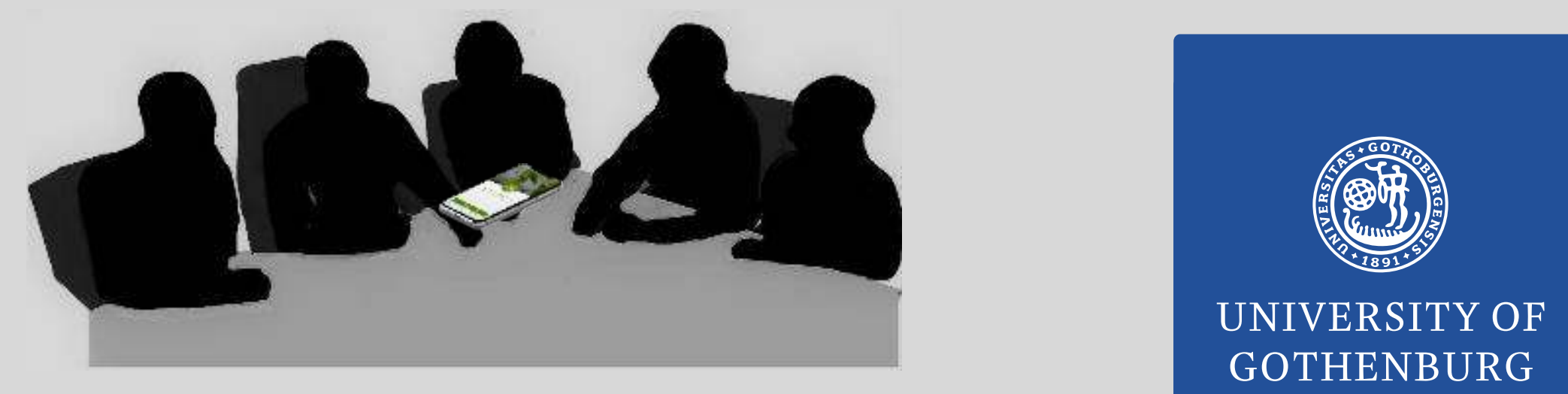